\title{
Recombinant Progranulin Prevents the Loss of Proteoglycan in Surgically Induced Osteoarthritis Model
}

\section{Chuanju Liu*}

Department of Orthopaedic Surgery and Cell Biology, New York University School of Medicine, 301 East 17th Street, New York, USA

\begin{abstract}
Osteoarthritis $(\mathrm{OA})$ is a degenerative joint disease that affects more than 46 million people in the United States alone. Since mechanisms by which OA ensues are largely unknown, there are no therapeutic targets that effectively prevent and treat the disease. However, growth factors, cytokines and matrix-degrading enzymes are strongly implicated in initiating and aggravating OA lesions. Thus, a molecular understanding of these molecules will provide invaluable information toward the search for novel therapeutic targets for OA. Our genome-wide screen for novel, differentially expressed genes in OA led to the isolation of progranulin (PGRN) as a novel OA-associated growth factor [1]. In subsequent global screen for the binding proteins of PGRN, we found that PGRN bound to TNF Receptors (TNFR). In addition, PGRN blocks the binding of TNFa to TNFR and inhibits TNFa-induced ADAMTS cleavage of cartilage oligomeric matrix protein (COMP) [2]. These previous findings led us to determine whether recombinant PGRN prevents cartilage degradation in the progression of OA in vivo. For this purpose, we took advantage of PGRN knockout mice to generate anterior cruciate ligament $(A C L)$ transection induced OA models which develop severe OA due to the deficiency of PGRN. Importantly, intra-articular injection of rPGRN growth factor significantly prevented the degeneration of cartilage in PGRN-deficient OA model. PGRN treated mice retained cartilage integrity and showed little or no degradation of cartilage matrix in comparison to highly degraded cartilage of non-treated mice (Figure)
\end{abstract}
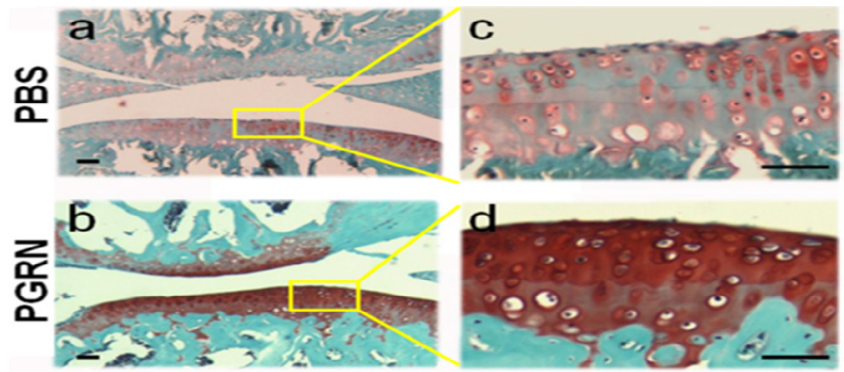

Figure 1: Recombinant progranulin (rPGRN) reduces the loss of proteoglycan in surgically induced osteoarthritis (OA) model. Intra-articular injection of rPGRN chondrogenic growth factor significantly prevented the degeneration of cartilage in PGRN-deficient OA model induced by anterior cruciate ligament $(A C L)$ transection. PGRN treated mice retained cartilage integrity and showed little or no degradation of cartilage matrix in comparison to highly degraded cartilage of non-treated mice.

\section{References}

1. Feng J, Guo F, Jiang B, Frenkel S, Zhang Y, et al. (2010), Granulin epithelin precursor: a bone morphogenic protein 2-inducible growth factor that activates Erk1/2 signaling and JunB transcription factor in chondrogenesis. FASEB J, $201024: 1879-1892$.

2. Tang W, Lu Y, Tian QY, Zhang Y, Guo FJ, et al. (2011) The Growth Factor Progranulin Binds to TNF Receptors and Is Therapeutic Against Inflammatory Arthritis in Mice, Science 332:478-484.
*Corresponding author: Chuanju Liu, Department of Orthopaedic Surgery and Cell Biology, New York University School of Medicine, 301 East 17th Street, New York, NY10003, USA, Tel: 212-598-6103; Fax: 212-598-6096; E-mail: Chuanju.Liu@ nyumc.org

Received October 31, 2014; Accepted October 31, 2014; Published November 01, 2014

Citation: Liu C (2014) Recombinant Progranulin Prevents the Loss of Proteoglycan in Surgically Induced Osteoarthritis Model. J Cytol Histol 5: i104. doi:10.4172/21577099.1000i104

Copyright: (C) 2014 Liu C. This is an open-access article distributed under the terms of the Creative Commons Attribution License, which permits unrestricted use, distribution, and reproduction in any medium, provided the original author and source are credited. 